\title{
An Automated System for Classification of Micro Calcification in Mammogram Based on Jacobi Moments
}

\author{
N. V. S. Sree Rathna Lakshmi and C. Manoharan
}

\begin{abstract}
Mammography is the only effective and viable technique to detect breast cancer especially in the case of minimal tumors. About $30 \%$ to $50 \%$ of breast cancers demonstrate deposits of calcium called micro calcifications. This paper presents an approach for detecting microcalcifications in mammograms employing Jacobi Moments. The set of Jacobi polynomials are orthogonal and this ensures minimal information redundancy between the moments. Jacobi moments encompass the properties of well known Zernike, Legendre and Tchebichef moments. Thus Jacobi moments are used as a feature set for the classification of mammogram as benign and malignant.
\end{abstract}

Index Terms-Jacobi Polynomials, Jacobi moments, micro calcifications

\section{INTRODUCTION}

Intensive research work has been undertaken in the development of automated image analysis methods to assist radiologists in the identification of abnormalities. The role computers play in mammogram analysis is threefold: detection, diagnosis and noise cancellation [1]. Detection involves identifying cancerous tissues in a mammogram. Early detection of breast cancer by mammography depends on the production of excellent images and competent interpretation. Many studies have been made on the problem of breast cancer diagnosing based on digital mammograms. Different methods have been used to classify and/or detect anomalies in medical images, such as wavelets [2,3], fractal theory [4], statistical methods [5] and most of them used features extracted using image-processing techniques [6]. In addition, some other methods were presented in the literature based on rule based fuzzy [7], Markov models [7], neural networks $[9,10]$ and fuzzy neural and feature extraction techniques [11]. Most of the computer-aided methods proved to be powerful tools that could assist medical staff in hospitals and lead to better results in diagnosing a patient.

Research into the detection of microcalcifications using the wavelet transform has been carried out in[3]. Given that the microcalcifications correspond to high frequency components of the image spectrum, detection of microcalcifications is achieved by decomposing the mammograms into different frequency sub-bands, suppressing the low-frequency sub-band, and, finally, reconstructing the mammogram from the sub-bands containing only high frequencies. The final images are obtained using sub-band reconstruction. They used two

Manuscript received January 10, 2011; revised May 12, 2011 wavelets from Daubechies' family namely the Daubechies' 4 (db 4) filter and Daubechies's 20 (db 20) filter.

According to the theory of deterministic fractal geometry, images can be modeled by deterministic fractal objects which are attractors of sets of two-dimensional (2-D) affine transformations. A methodology based on fractal image modeling is developed to analyze and model breast background structures in [4] and microcalcifications can be enhanced by taking the difference between the original image and the modeled image. This research showed that the fractal modeling method is an effective way to enhance microcalcifications.

Markov random field (MRF) model based method is described in [8]. The detection of tumors is performed in two steps: segmentation and classification. In segmentation, regions of interest are first extracted from the images by adaptive thresholding. A system based on fuzzy-neural and feature extraction techniques for detecting and diagnosing microcalcifications' patterns in digital mammograms is developed in [11]. The back-propagation technique was used for classification of features into benign or malignant

Moment based feature descriptors have evolved into a powerful tool for image analysis applications. Geometric moments present a low computational cost, but are highly sensitive to noise. Furthermore reconstruction is extremely difficult. Although not invariant under rotation, Hu's invariants [12] that are derived from geometric moments present invariance under linear transformations. Complex moments provide with additional invariant descriptors, but present the same problems regarding noise and reconstruction.

Global geometric moments and their invariants are widely used in many areas of image analysis, including pattern recognition [12], image reconstruction [13], and fingerprint Recognition [14]. In addition to geometric moments, which are also known as regular or ordinary moments, a number of other moments have been proposed. The notion of complex moments was introduced in [15] for deriving moment invariants. Teague suggested the use of orthogonal moments and introduced complex valued Zernike moments that are defined on a unit disk. A second class of orthogonal moments is given by Legendre moments which make use of Legendre polynomials. The usefulness of Legendre and Zernike moments has been demonstrated, in particular, for image reconstruction [13], [16] and pattern classification [17]. The pseudo-Zernike formulation proposed in [18] further improved these characteristics.

In this class, Legendre and Zernike moments have been extensively researched in the recent past, and several new techniques have emerged involving orthogonal moment 
based feature detectors [19], [20].

\section{JACOBI POLYNOMIALS}

The kernel of the Jacobi moments consists of the set of Jacobi polynomials. The Jacobi polynomial of the $\mathrm{n}^{\text {th }}$ order, with parameter $\alpha$ and $\beta$, is defined as.

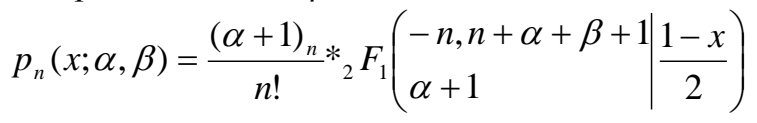

where $x \in[-1,1]$. The generalized hyper geometric function,

${ }_{r} F_{s}$, is defined as:

$$
{ }_{r} F_{s}\left(\begin{array}{l}
a_{1}, \ldots a_{r} \\
b_{1}, \ldots b_{r}
\end{array} \mid z\right)=\sum_{k=0}^{\infty} \frac{\left(a_{1}, \ldots a_{r}\right) k}{\left(b_{1}, \ldots b_{r}\right) k} \frac{z^{k}}{k !}
$$

where $\left(a_{1}, \ldots a_{r}\right) k=\left(a_{1}\right) k, \ldots\left(a_{r}\right) k$ and the Pochhammer-symbol:

$$
(a) k=a(a+1)(a+2) \ldots(a+k-1)
$$

with $\mathrm{k}=1,2,3, \ldots$ and $(a)_{0}=1$ and thus have the explicit expression

$$
\rho(n ; \alpha, \beta)=\frac{2^{\alpha+\beta+1}}{2 n+\alpha+\beta+1} * \frac{\Gamma(n+\alpha+1) \Gamma(n+\beta+1)}{\Gamma(n+\alpha+\beta+1) n !}
$$

The Jacobi polynomials can be written in the Rodrigues-type formula as:

$$
(1-x)^{\alpha}(1+x)^{\beta} P_{n}(x ; \alpha, \beta)=\frac{(-1)^{n}}{2^{n} n !}\left(\frac{d}{d x}\right)^{n}\left[(1-x)^{n+\alpha}(1+x)^{n+\beta}\right]
$$

For $\alpha>-1, \beta>-1$, the set of Jacobi polynomials satisfy the orthogonality condition:

$$
\int_{-1}^{1} \omega(x ; \alpha, \beta) P_{m}(x ; \alpha, \beta) P_{n}(x ; \alpha, \beta) d x=\rho(n ; \alpha, \beta) \delta_{m, n},
$$

where $\delta_{m n}$ is the Kronecker delta and

$$
\begin{gathered}
\omega(x ; \alpha, \beta)=(1-x)^{\alpha}(1+x)^{\beta}, \\
\rho(n ; \alpha, \beta)=\frac{2^{\alpha+\beta+1}}{2 n+\alpha+\beta+1} * \frac{\Gamma(n+\alpha+1) \Gamma(n+\beta+1)}{\Gamma(n+\alpha+\beta+1) n !}
\end{gathered}
$$

where $\Gamma($.$) being the gamma function.$

\section{JACOBI MOMENTS}

The Jacobi moment of order $(p+q)$ of an image $f(x, y)$ with $\mathrm{Mx} \mathrm{N}$ pixels is defined as:

$$
J_{m n}=\sum_{x=0}^{M-1} \sum_{y=0}^{N-1} f(x, y) \bar{P}_{m}(x) \bar{P}_{n}
$$

where

$$
\begin{aligned}
& \overline{P_{m}}(x) \equiv \overline{P_{m}}\left(x ; \alpha_{1}, \beta_{1}\right), \\
& \bar{P}_{n}(y) \equiv \bar{P}_{n}\left(y ; \alpha_{2}, \beta_{2}\right),
\end{aligned}
$$

and

$$
\bar{P}_{n}(x ; \alpha, \beta)=P_{n}(x ; \alpha, \beta) \sqrt{\frac{\omega(x ; \alpha, \beta)}{\rho(n ; \alpha, \beta)}},
$$

that:

$$
\sum_{x=0}^{N-1} \bar{P}_{m}(x ; \alpha, \beta) \bar{P}_{n}(x ; \alpha, \beta)=\delta_{m n}
$$

The term $\bar{P}_{n}($.$) is the weighted Jacobi polynomials.$

\section{PRoposed SYSTEM}

The block diagram of the proposed mammogram classification system is shown in Figure 1, where Figure 1(a) depicts the Feature Extraction Phase while classification Phase is shown in the Figure 1(b).
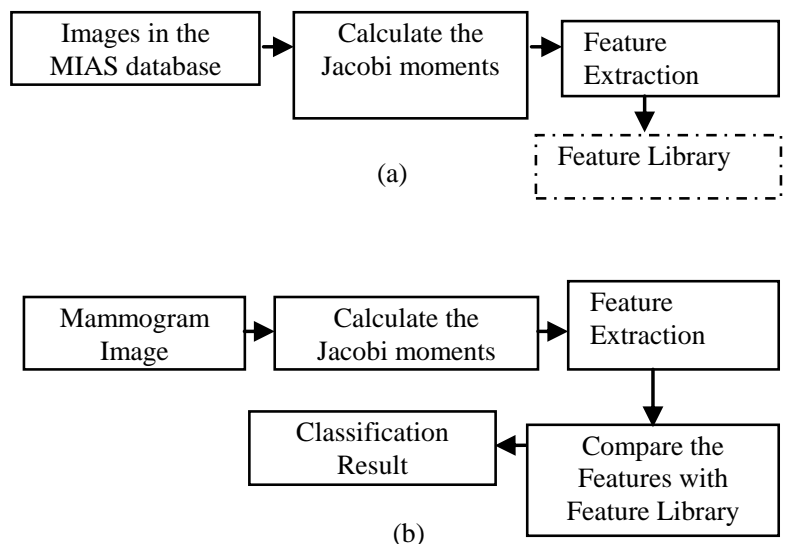

(a) Feature Extraction Phase (b) Classification Phase Figure1.Proposed mammogram classification system

\section{A. Feature Extraction}

Feature extraction is an essential pre-processing step to pattern recognition and machine learning problems. It is often decomposed into feature construction and feature selection. In our approach, Jacobi moments are used as a feature to classify the mammogram images .In the following section gives the overview of feature extraction of the known microcalcification area in the digital mammogram.

The input to the feature extraction module is the well known microcalcification area in the MIAS database mammogram images. The location of the microcalcification area in the digital mammogram is given by the MIAS database. First, the known microcalcification area is separated from the whole image and the Jacobi moments are calculated for the mass area only. A 4X4 overlapping window is used to extract the Jacobi moments. By carefully analyzing the moment's values for all the images, a range is selected for each order as a feature set.

Fig (1). (a) Shows a digital mammogram from the MIAS database. (mdb223.pgm) (b) Location of the microcalcification in the whole image. The Jacobi moments are calculated for the microcalcification area only.

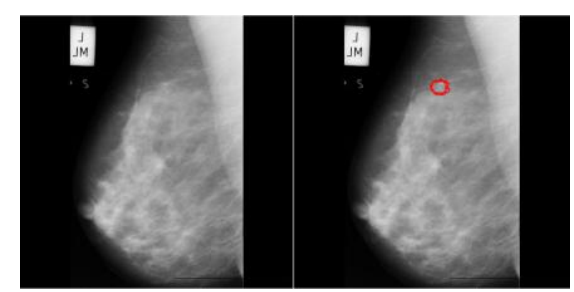

(a)

(b)

Figure 2. (a) MIAS database image (mdb223) (b) Location of the Microcalcification area in the whole image 


\section{B. Jacobi moments Calculation}

Jacobi moments for all overlapping window size of $4 \mathrm{X} 4$ are calculated. The moment's value for each order is plotted and the limit is calculated. A set of 10 abnormal images are selected from the MIAS database and all the limits are calculated. From the 10 limits for each order, new limit is selected as a feature set for that order.

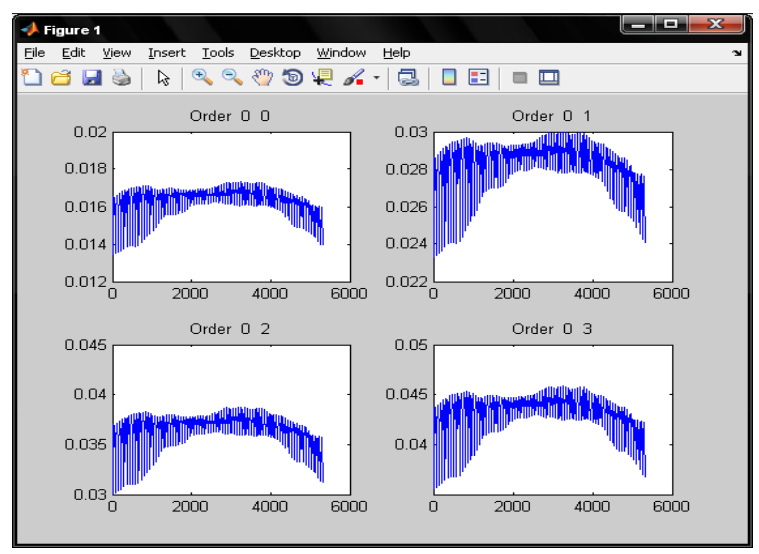

Figure 3. Plot of Jacobi moments for each order for the limit calculation

Then the limit is used as a feature vector to classify the unknown image as normal or abnormal as well as benign or malignant. The following figure shows the plot of moments order for MIAS database image. (mdb028.pgm). Finally, the feature set for abnormal, benign and malignant images are calculated.

\section{Pseudo Code}

The Pseudo code for the calculation of Jacobi moments are given below.

\section{BEGIN JACOBIMOMENTS () \\ STEP 1. INITIALIZATION}

Input: Image $f(x, y)$ with $\mathrm{M} x \mathrm{~N}$ pixels

Define MAXORDER

Define $\alpha_{1}, \alpha_{2}, \beta_{1}, \beta_{2}$

\section{STEP 2. MOMENTS CALCULATON}

For each order

Compute the following

$$
\bar{P}_{m}(x)=\text { JACOBIPOLYNOMIAL (m, x, } \alpha_{1}, \beta_{1} \text { ) }
$$$$
\bar{P}_{n}(x)=\text { JACOBIPOLYNOMIAL (n, } \mathrm{x}, \alpha_{2}, \beta_{2} \text { ) }
$$

Calculate $I_{\operatorname{mn}}$ using (7).

\section{END JACOBIMOMENTS ()}

\section{BEGIN JACOBIPOLYNOMIAL $\left(\boldsymbol{m}, \boldsymbol{x}, \boldsymbol{\alpha}_{\mathbf{1}}, \boldsymbol{\beta}_{\mathbf{1}}\right)$}

1. Calculate $\omega\left(x, \alpha_{1}, \beta_{1}\right)$ using $(7)$

2. Calculate $\rho\left(n, \alpha_{1}, \beta_{1}\right)$ using (7)

3. Calculate $P_{n}\left(x ; \alpha_{1}, \beta_{1}\right)$ using (4)

4. Calculate $\bar{P}_{n}\left(x_{;} \alpha_{x} \beta\right)$ using (10)

Return

END JACOBIPOLYNOMIAL ()

\section{EXPERIMENTAL RESULTS}

The experiments are conducted on the MIAS database [21]. In MIAS database, there are 194 normal images and 25 micro calcification images available. All the images are considered for the classification test. The number of training samples used for the limit calculation and testing images for classification is given in Table 1. The classification rate obtained using Jacobi feature sets results are show in Table 2.

TABLE.1. NUMBER OF TRAINING AND TESTING SAMPLES

\begin{tabular}{|c|c|c|}
\hline $\begin{array}{c}\text { Type of } \\
\text { image }\end{array}$ & $\begin{array}{c}\text { No of training } \\
\text { Images }\end{array}$ & $\begin{array}{c}\text { No of Testing } \\
\text { Images }\end{array}$ \\
\hline Normal & 30 & 194 \\
\hline Abnormal & 12 & 25 \\
\hline Benign & 6 & 12 \\
\hline Malignant & 6 & 13 \\
\hline
\end{tabular}

TABLE.2. CLASSIFICATION RESULT OF THE PROPOSED SYSTEM

\begin{tabular}{|c|c|}
\hline Type of image & $\%$ Classification rate \\
\hline Normal & 87.63 \\
\hline Abnormal & 84.00 \\
\hline Benign & 83.33 \\
\hline Malignant & 84.62 \\
\hline
\end{tabular}

\section{CONCLUSION}

This paper presents a method for breast tumor detection in mammograms. Our method is based on Jacobi Moments and is capable of detecting suspicious areas in mammograms independent of their size, orientation, and position. Our method is successful in detecting the micro calcification in the mammogram. To assess its clinical utility, it has to be tested on much larger sets of data. Furthermore, a clinically useful system for breast cancer detection must be able to detect the breast cancers, not just micro calcifications. Our future work is to extend the Jacobi moment feature for other type of mammogram.

\section{REFERENCES}

[1] Kristin J. McLoughlin, Philip J. Bones and Nico Karssemeijer, "Noise Equalization for Detection of Microcalcification Clusters in Direct Digital Mammogram Images" IEEE Trans. Medical Imaging, vol. 23 pp. 313-320,March.2004.

[2] Zhongchao and Ryosuke," An approach to image segmentation using multiresolution analysis of Wavelets" International Journal of Pattern Recognition and Artificial Intelligence, 7(6):810-815, 1999

[3] T.Wang and N. Karayiannis. "Detection of micro calcification in digital mammograms using wavelets". IEEE Trans. Medical Imaging, 17(4):498-509, 1998.

[4] H. Li et al. "Fractal modeling and segmentation for the enhancement of microcalcifications in digital mammograms". IEEE Trans. Medical Imaging, 16(6):785-798, 1997.

[5] H. Chan et al. Computerized analysis of mammographic microcalcifications in morphological and feature spaces. Medical Physics, 25(10):2007-2019, 1998.

[6] S. Lai, X. Li, and W. Bischof. On techniques for detecting circumscribed masses in mammograms. IEEE Trans. Medical Imaging, 8(4):377-386, 1989.

[7] D. Brazokovic and M. Neskovic. Mammogram screening using multi resolution-based image segmentation. International Journal of Pattern Recognition and Artificial Intelligence, 7(6):1437-1460, 1993.

[8] H. Li et al. Marcov random field for tumor detection in digital mammography. IEEE Trans. Medical Imaging, 14(3):565-576, 1995.

[9] I. Christoyianni et al. Fast detection of masses in computer-aided mammography. IEEE Signal Processing Magazine, pages 54-64, Jan 2000.

[10] A. Dhawan et al. Radial-basis-function-based classification of mammographic microcalcifications using texture features. In Proc. of the 17th Annual International Conference of the IEEE Engineering in Medicine and Biology Society, volume 1, pages 535- 536, 1995.

[11] Brijesh Verma and John Zakos , "A Computer-Aided Diagnosis System for Digital Mammograms Based on Fuzzy-Neural and Feature Extraction Techniques" IEEE Trans on nformation technology in biomedicine, vol. 5, pp 46-54, March 2001. 
[12] M.-K. Hu, "Visual pattern recognition by moment invariants," IRE Trans. Inform. Theory, pp. 179-187, Feb. 1962.

[13] C. Teh and R. Chin, "On image analysis by the methods of moments," IEEE Trans. Pattern Anal. Machine Intell., vol. 10, pp. 496-513, July 1988.

[14] Hasan Abdel Qader,and Syed Al-Haddad,"Fingerprint Recognition using Zernike moments", The International Arab journal of information technology, vol 4, Oct 2007.

[15] Y. Abu-Mostafa and D. Psaltis, "Recognitive aspects of moment invariants," IEEE Trans. Pattern Anal. Machine Intell., vol. PAMI-6, pp. 698-706, Nov. 1984

[16] S. Liao and M. Pawlak, "On image analysis by moments," IEEE Trans. Pattern Anal. Machine Intell., vol. 18, pp. 254-266, Mar. 1996.
[17] A. Khotanzad and Y. Hong, "Invariant image recognition by zernike moments," IEEE Trans. Pattern Anal. Machine Intell., vol.48, pp. 489-497, May 1990.

[18] Mirosław Pawlak, and Simon X. Liao,"On the Recovery of a Function on a Circular Domain". IEEE Trans. Pattern Anal. Machine Intell., vol. 12, pp. 2736-2755, Oct 2002.

[19] Pew-Thian Yap and Raveendran paramesram,"Jacobi Moments as Image Features". International Journal of Pattern Recognition and Artificial Intelligence, 7(6):594-597, 2004

[20] Pew-Thian Yap and Seng-Huat Ong," Image Analysis Using Hahn Moments", IEEE Trans. Pattern Anal. Machine Intell., vol. 11, pp. 2057-2062, Nov 2007.

[21] MIAS database, www.mias.org 